BULL. AUSTRAL. MATH. SOC.

$46699,46 A 05$

VOL. 24 (1981), 69-77.

\title{
MEAN VALUE THEOREMS AND A TAYLOR THEOREM FOR VECTOR VALUED FUNCTIONS
}

\author{
RUDOLF VÝBORNÝ
}

\begin{abstract}
Two mean value theorems and a Taylor theorem for functions with values in a locally convex topological vector space are proved without the use of the Hahn-Banach extension theorem.
\end{abstract}

\section{Introduction}

It is well known that the classical mean value theorem does not hold for vector valued functions. For example, if $f(x)=e^{i x}$, $x \in[a, b]=[0,2 \pi]$ then the equation

$$
f(b)-f(a)=f^{\prime}(c)(b-a)
$$

cannot hold for any $c \in(0,2 \pi) .\left(f(b)=f(a),\left|f^{\prime}(c)\right|=1\right.$ for every $c \in(a, b)$.$) In the mathematical literature there appear two kinds of$ valid generalization of the mean value theorem for vector valued functions.

(a) The equation (1) is replaced by an inequality involving norms or seminorms. For complex valued function this goes back to Darboux [3] who proved: if $f:[a, b] \rightarrow \mathbf{C}$ has a continuous derivative then there exists a $c \in(a, b)$ such that

$$
|f(b)-f(a)| \leq\left|f^{\prime}(c)\right|(b-a) .
$$

For linear normed spaces this generalization without the assumption of continuity of $f^{\prime}$ is due to $A z i z$ and Diaz [1], the sign $|\cdot|$ in inequality (2) in this case must be interpreted as a norm. For further

Received 17 February 1981. This research was partly supported by a grant from Deutscher Akademischer Austauschdienst. The author wishes to express his gratitude to W.F. Eberlein for making available his paper [8]. 
development see [2], [4], and for history of the mean value theorem [10]. (Also concerning generalizations (b) to follow.)

(b) The equation (1) is replaced by the statement that $\frac{f(b)-f(a)}{b-a}$ lies in the closed convex hull of the range of the derivative. In the sequel, the convex hull of $A$ shall be denoted by $\operatorname{co} A$ and a closure by a bar. Hence

$$
\frac{f(b)-f(a)}{b-a} \in \overline{\operatorname{co}}\left\{f^{\prime}(t) ; a<t<b\right\} .
$$

This generalization for complex valued functions goes back to Weierstrass [12, p. 58], for linear normed spaces is due to Wazewski [16]. There is a nice example by Eberlein [8] showing that the bar cannot be omitted in (3) in case of an infinite dimensional space. See also [17]. McLeod [13] proved that the bar can be omitted if the space is finite dimensional. For locally convex spaces see [6] and [13].

There is another feature to these generalizations, namely, the occurrence of an exceptional set $E \subset(a, b)$ where the function is either not required to be differentiable or the values of the derivative are not taken into account (McLeod [13] actually works with two exceptional sets). The classical mean value theorem is no longer true even if $E$ is a singleton; however, in both generalizations mentioned above some infinite sets are permitted.

There is yet another feature common to both generalizations, namely, most of the proofs (an exception is, for example, [4]) employ the HahnBanach theorem. It is one of the aims of this paper to prove a Cauchy mean value theorem of Weierstrass' type for functions with values in a locally convex topological vector space. By avoiding the Hahn-Banach Theorem and hence also the axiom of choice (and by not using Solovey's axiom either) we obtain a theorem of constructive functional analysis in the sense of Garnir [11].

In a certain sense Weierstrass' mean value theorem is stronger than Darboux mean value theorem [8], [15].

There exist very many generalizations of the Taylor theorem to vector valued functions. Besides the integral form of the remainder (see, for example, [7], [14, p. 188]) which is most often used there also exist 
estimates of the remainder similar to Darboux type generalizations of the Mean Value Theorem [5], [9, Theorem 3]. In this paper we prove an estimate of the remainder in the spirit of Weierstrass generalization of the Mean Value Theorem. The advantage of our approach lies in the fact that we use only the mere existence of the $(n+1)$ st derivative.

\section{Mean Value Theorems}

LEMMA. If $\delta:[a, b] \rightarrow R_{+}$(that is, $\delta$ is real valued and positive), $M \subset[a, b], G=\left\{\left(\alpha_{i}, \beta_{i}\right)\right\}$ is a family (finite or infinite) of disjoint open intervals, $\left[\alpha_{i}, \beta_{i}\right] \subset[a, b]$ and if

$$
[a, b] \subset \underset{\xi \in M}{\cup}(\xi-\delta(\xi), \xi+\delta(\xi)) \cup \underset{i}{\cup}\left(\alpha_{i}, \beta_{i}\right)
$$

then there exists a partition (P) of $[a, b]$,

$$
a=x_{0}<x_{1}<x_{2}<\ldots<x_{n}=b
$$

with the following property: for every $j, j=0,1, \ldots, n-1$, either $\left(x_{j}, x_{j+1}\right)$ is one of the intervals $\left(\alpha_{i}, \beta_{i}\right) \in G$ or there is a $\xi_{j} \in M \cap\left[x_{j}, x_{j+1}\right]$ and

$$
\begin{gathered}
x_{j+1}-\xi_{j}<\delta\left(\xi_{j}\right), \\
\xi_{j}-x_{j}<\delta\left(\xi_{j}\right) .
\end{gathered}
$$

The latter intervals will be referred to as $\xi$-intervals.

Proof. If $G$ is empty an indirect proof based on bisecting $[a, b]$ and on the nested interval theorem is easy. The general case can be reduced to the case of finitely many intervals for which $G$ is empty. This is done by applying Borel's covering theorem to the system of open intervals consisting of intervals of $G$ and of intervals $(\xi-\delta(\xi), \xi+\delta(\xi)), \xi \in M$.

THEOREM 1. If

(i) $X$ is a real locally convex topological vector space,

(ii) $f:[a, b] \rightarrow X$ is continuous at $a$ and $b$ from the right and left respectively, 
(iii) $g:[a, b] \rightarrow R$ is strictly increasing, continuous at a and $b$, from the right and from the left, respectively,

(iv) $E$ is a subset of $(a, b)$ and $f$ and $g$ are differentiable on $(a, b)-E$; moreover $g^{\prime}$ is positive on $(a, b)-E$,

(v) $C$ is convex and closed, $\left\{\frac{f^{\prime}(t)}{g^{\prime}(t)} ; t \in(a, b)-E\right\} \subset c$,

(vi) for every neighbourhood of zero $U$ there exists a family of disjoint open intervals $\left(\alpha_{i}, \beta_{i}\right)$ such that

$$
E \subset U_{i}\left(\alpha_{i}, \beta_{i}\right) \text {, }
$$

and for any finite set of i's,

$$
\sum\left[f\left(\beta_{i}\right)-f\left(\alpha_{i}\right)\right] \in U,
$$

(vii) $0 \in C$,

then

$$
\frac{f(b)-f(a)}{g(b)-g(a)} \in C
$$

Proof. Denote $q=\frac{f(b)-f(a)}{g(b)-g(a)}$ and let $V$ be a convex, symmetric neighbourhood of zero. The theorem will be proved if we show that there exists an element $c \in C$ such that $q-c \in V$. There exist positive $\delta(\alpha)$ and $\delta(b)$ such that

$$
f(x)-f(a) \in \frac{g(b)-g(a)}{4} \cdot V
$$

and

$$
f(x)-f(b) \in \underline{g(b)}-g(\underline{a}) \cdot V
$$

for $a \leq x \leq a+\delta(a)$ and $b-\delta(b) \leq x \leq b$, respectively. Further, for every $\xi \in(a, b)-E$ there exists a positive $\delta(\xi)$ such that

$$
\frac{f(x)-f(\xi)}{g(x)-g(\xi)}-\frac{f^{\prime}(\xi)}{g^{\prime}(\xi)} \in \frac{1}{4} V
$$

for $0<|x-\xi| \leq \delta(\xi)$. Let $\left(\alpha_{i}, \beta_{i}\right)$ be the system of intervals from 
(vi). Let (P) be the partition from Lemma for $M=(a, b)-E$. If $\left(x_{j}, x_{j+1}\right)$ is a $\xi$-interval then it follows from (8) using strict monotonicity of $g$ and convexity of $V$ that

$$
\frac{f\left(x_{j+1}\right)-f\left(x_{j}\right)}{g\left(x_{j+1}\right)-g\left(x_{j}\right)}-\frac{f^{\prime}\left(\xi_{j}\right)}{g^{\prime}\left(\xi_{j}\right)} \in \frac{1}{4} V .
$$

We denote by $\sum^{\prime}$ the sum which is extended over those $j$ for which $\left(x_{j}, x_{j+1}\right)$ is a $\xi$-interval and in $\Sigma^{\prime \prime}$ the summation is understood over the remaining indices. We define $\lambda_{j}=\frac{g\left(x_{j+1}\right)-g\left(x_{j}\right)}{g(b)-g(a)}, c=\sum^{\prime} \lambda_{j} \frac{f^{\prime}\left(\xi_{j}\right)}{g^{\prime}\left(\xi_{j}\right)}$ (if there is no $\xi$-interval in (P) we define $c=0$ ). Since $0 \leq \lambda_{i} \leq 1$, $\sum^{\prime} \lambda_{i} \leq 1$ we have, by using (vii), that $c \in C$. Now we estimate $q-c$;

$$
\begin{aligned}
q-c & =\sum^{\prime} \lambda_{j} \frac{f\left(x_{j+1}\right)-f\left(x_{j}\right)}{g\left(x_{j+1}\right)-g\left(x_{j}\right)}-c+\frac{1}{g(b)-g(a)} \sum^{\prime \prime}\left[f\left(x_{j+1}\right)-f\left(x_{j}\right)\right] \\
& \in \frac{1}{4} V+\frac{3}{4} V,
\end{aligned}
$$

by using (vi) with $U=\frac{1}{4} V,(7)$ and (9). Hence we established $q-c \in V$ and the theorem is proved.

REMARK 1. The hypothesis $0 \in C$ cannot be omitted without affecting the validity of Theorem 1 . For a counterexample see [6, Remark 0, pp. 295-296].

However, if the hypothesis $(v)$ of Theorem 1 is replaced by the following stronger hypothesis,

(vis) $E$ is a subset of $(a, b)$ such that, for every $\varepsilon>0$ and every neighbourhood of zero $U$, there is a family of disjoint open intervals $\left(\alpha_{i}, \beta_{i}\right),\left[\alpha_{i}, \beta_{i}\right] \subset[a, b]$ such that (5), (6) hold and moreover

$$
\sum\left[g\left(B_{i}\right)-g\left(\alpha_{i}\right)\right]<\varepsilon ;
$$

then the hypothesis (vii) can be omitted. More precisely, we have

THEOREM 2. If hypotheses $(i)-(v)$ and (vis) hold then 


$$
\frac{f(b)-f(a)}{g(b)-g(a)} \in \overline{c o}\left\{\frac{f^{\prime}(t)}{g^{\prime}(t)} ; t \in(a, b)-E\right\} \text {. }
$$

Proof. We keep the notation from the proof of Theorem $1,\left(\alpha_{i}, B_{i}\right)$ now satisfy (vis) rather than (vi). We choose a positive $\varepsilon$ satisfying $\varepsilon<\frac{1}{2}\{g(b)-g(a)\}, \frac{2 \varepsilon}{\{g(b)-g(a)\}} q \in V$. It follows that $\sum^{\prime} \lambda_{j} \geq \frac{1}{2}$, in particular there are $\xi$-intervals and $c=\sum^{\prime} \lambda_{j} \frac{f^{\prime}\left(\xi_{j}\right)}{g^{\prime}\left(\xi_{j}\right)}$. Define $a=\sum^{\prime} \lambda_{j}, d=\frac{1}{a} c$. Then $d \in \operatorname{co}\left\{\frac{f^{\prime}(\xi)}{g^{\prime}(\xi)} ; \xi \in(a, b)-E\right\}$. Finally, we have $q-d=q-\frac{1}{a} q+\frac{1}{a}(q-c) \in V+\frac{1}{a} V \subset 3 V$. This completes the proof.

REMARK 2. Some examples when hypothesis (viS) is satisfied are:

(a) $f$ and $g$ continuous throughout $[a, b]$ and $E$ is at most countable;

(b) $\because$ and $g$ are absolutely continuous and $E$ is of measure zero;

(c) $f$ is absolutely continuous with respect to $g$ and $E$ is of $g$-measure zero;

(d) $f$ is $\alpha$-Hölder continuous, $g(x)=x$, and $E$ is of $\alpha$-Hausdorff measure zero.

REMARK 3. One can obtain a mean value theorem for a mapping $F$ from one locally convex topological vector space to another by using either Theorem 1 or Theorem 2 on the function $f$ of a real variable defined by $f(t)=F(a+t(b-a))$.

\section{Taylor's Theorem}

Let $X$ and $Y$ be locally convex topological spaces, $a \in X$, $a+x \in X, f: X \rightarrow Y, n+1$ times Gateaux differentiable at all points of the segment $[a, a+x]$. Let further $g:[0,1] \rightarrow R$ be continuous and $g^{\prime}(t)>0$ for $0<t<1$. Then 
(11) $f(a+x)-\sum_{i=0}^{n} f^{(i)}(a)\left(x^{i}\right)$

$$
\epsilon \frac{g(1)-g(0)}{n !} \operatorname{co}\left\{\frac{(1-t)^{n} f^{n+1}(a+t x)\left(x^{n+1}\right)}{g^{\prime}(t)}, 0<t<1\right\} .
$$

In particular, for $g(t)=-(1-t)^{n}$,

(12) $f(a+x)-\sum_{i=0}^{n} f^{(i)}(a)\left(x^{i}\right) \in \operatorname{co}\left\{\frac{f^{(n+1)}(a+t x)\left(x^{n+1}\right)}{(n+1) !}, 0<t<1\right\}$.

REMARK 4. For convenience of exposition we assumed the existence of $f^{(n+1)}(a+t x)$ for all $t \in[0,1]$. One can, however, weaken this hypothesis and allow an exceptional set $E$, similarly as in paragraph 2 .

Proof. Let us define

$$
H(t)=f(a+t x)+\frac{(1-t)}{1 !} f^{\prime}(\alpha+t x)(x)+\ldots+\frac{(1-t)^{n}}{n !} f^{(n)}(a+t x)\left(x^{n}\right) .
$$

An easy calculation shows $H^{\prime}(t)=\frac{(1-t)^{n}}{n !} f^{(n+1)}(\alpha+t x)\left(x^{n+1}\right)$. Applying the mean value theorem to $H, g$ and the interval $[0,1]$, we obtain (11). The relation (12) is then immediate.

\section{References}

[1] A.K. Aziz and J.B. Diaz, "On a neam-value theorem of the dirferential calculus of vector-valued functions, and uniqueness theorems for ordinary differential equations in a linear-normed space", Contrib. Differential Equations 1 (1963), 251-269.

[2] A.K. Aziz, J.B. Diaz and W. Mlak, "On a mean value theorem for vector-valued functions, with applications to uniqueness theorems for 'right-hand-derivative' equations", J. Math. Anal. App Z. 16 (1966), 302-307.

[3] G. Darboux, "Sur les développments en série des fonctions d'une seule variable", Liouvilze J. (3) 11 (1876), 291-312. 
[4] J.B. Diaz and R. Výborný, "On mean value theorems for strongly continuous vector valued functions", Contrib. Differential Equations 3 (1964), 107-118.

[5] Joaquin B. Diaz and R. Výborný, "A fractional mean value theorem, and a Taylor theorem, for strongly continuous vector valued functions", Czechoslovak Math. J. 15 (90) (1965), 299-304.

[6] J.B. Diaz and R. Výborný, "Generalized mean value theorems of the differential calculus", J. Austral. Math. Soc. Ser. A 20 (1975), 290-300.

[7] J. Dieudonné, Foundations of modern analysis (Pure and Applied Mathematics, 10. Academic Press, New York and London, 1960).

[8] W.F. Eberlein, "The mean value theorem for vector valued functions", preprint.

[9] T.M. Flett, "Mean value theorems for vector-valued functions", Tôhoku Math. J. 24 (1972), 141-151.

[10] T.M. Flett, "Some historical notes and speculations concerning the mean value theorems of the differential calculus", Bull. Inst. Math. Appl. 10 (1974), no. 3, 66-72.

[11] H.G. Garnir, "Solovay's axiom and functional analysis", Functional analysis and its applications, 189-204 (Proc. Internat. Conf., Madras, 2973. Lecture Notes in Mathematics, 399. SpringerVerlag, Berlin, Heidelberg, New York, 1974 ).

[12] Ch. Hermite, Cours de la Faculté des Sciences de Paris sur Zes intégrales définies, la théorie des fonctions d'une variable imaginaire, et les fonctions elziptiques, 4 éa., entièrement refondue (Rédigé par M. Andoyer. Hermann, Paris, 1891).

[13] Robert M. Mcleod, "Mean value theorems for vector valued functions", Proc. Edinburgh Math. Soc. (2) 14 (1964-1965), 197-209. 
[14] M.Z. Nashed, "Differentiability and related properties of nonlinear operators: some aspects of the role of differentials in nonlinear functional analysis", Nonlinear functional analysis and applications, 103-309 (Proc. Advanced Seminar, Mathematics Research Center, University of Wisconsin, Madison, 1970. Publication No. 26 of the Mathematics Research Center, The University of Wisconsin. Academic Press, New York, London, 1971).

[15] Jacek Szarski, Differential inequalities (Monografie Matematiyczne, 43. PWN - Polish Scientific Publishers, Warszawa, 1965).

[16] T. Wazewski, "Une généralisation des théorèmes sur les accroissements finis au cas des espaces de Banach et application à la généralisation du théorème de l'Hôpital", Ann. Soc. Polon. Math. 24 (1951), no. 2, 132-147 (1954).

[17] Sadayuki Yamamuro, Differential calculus in topological linear spaces Lecture Notes in Mathematics, 374. Springer-Verlag, Berlin, Heidelberg, New York, 1974).

Department of Mathematics, University of Queensland, St Lucia, Queensland 4067, Australia. 